\title{
Figure of merit for the divertor protection in the preliminary design of EU-DEMO reactor
}

\author{
M. Siccinio ${ }^{1,2^{*}}$, G. Federici ${ }^{1}$, R. Kembleton ${ }^{1,3}$, H. Lux ${ }^{3}$, F. Maviglia ${ }^{1,4}$, J. Morris $^{3}$ \\ ${ }^{1}$ PPP\&T Department, EUROfusion Consortium, Boltzmannstr. 2, 85748 Garching bei München, Germany \\ ${ }^{2}$ Max-Planck-Institut für Plasmaphysik, , Boltzmannstr. 2, 85748 Garching bei München, Germany \\ ${ }^{3}$ EURATOM/CCFE Fusion Association, Culham Science Centre, Abingdon, Oxon, OX14 3DB, United Kingdom \\ ${ }^{4}$ Associazione EURATOM ENEA, Frascati, Rome, Italy
}

${ }^{*}$ Corresponding author: mattia.siccinio@euro-fusion.org

\begin{abstract}
This paper discusses the criteria to be used in the preliminary design phases of the EU-DEMO reactor to ensure the performance of the divertor without compromising the stability of core plasma or the fusion power generation. This work refers to a lower single null conventional divertor using actively cooled solid metal plasma-facing components and with extrinsic seeding for heat flux dissipation, which is the solution currently adopted for EUDEMO. The analysis does not consider the role of the Edge Localised Modes (ELMs), and also neglects major offnormal events like disruptions. It is shown that it is necessary to fulfil two high-level requirements, namely: i) the concentration of seeded impurities has to be lower than some critical value in order not to compromise the fusion plasma performance or stability and ii) damage to the divertor plate in case of accidental plasma reattachment must be avoided for a time sufficiently long to ensure a safe, controlled termination of the plasma discharge, as, in a device like EU-DEMO, other strategies relying on mass injection are considered more likely to cause a loss of plasma stability at full current, with dramatic consequences for the integrity of plasma facing components. Two figures of merit, corresponding to these criteria, have been identified in the existing literature and discussed. The dependence of such figures of merit on the relevant machine parameters (major radius and toroidal magnetic field) is analysed. Initially, the analysis is carried out using a simple OD physics approach and subsequently by means of the systems code PROCESS, which allows for consideration of further parameters, such as aspect ratio and elongation. The main conclusion of the present work is that the simultaneous fulfilment of both requirements limits the viable EU-DEMO size both in terms of major radius $R$ and in terms of toroidal magnetic field $B_{T}$. Finally, an attempt to extend the EU-DEMO related conclusions to a more general level is made.
\end{abstract}

\section{Introduction}

To achieve satisfactory performance in terms of net electric power output, tokamak fusion reactors have to possess adequately large magnetic fields $\left(B_{T}\right)$ and size (in terms of major radius, $R$ ) in order to obtain a sufficient particle and energy confinement time for the burning plasma. The European DEMOnstration reactor EU-DEMO [1], for example, possesses a major radius $R$ of around $9 \mathrm{~m}$ and is operated with a magnetic field on the axis, $B_{T}$, of around $6 \mathrm{~T}$. This yields a fusion power $P_{f u s}$ of $2 \mathrm{GW}$ if the confinement time $\tau_{E}$ obeys the widely used IPB98 $(\mathrm{y}, 2)$ scaling [2]. Such a level of fusion power is necessary in order to meet the requirement of producing a few hundred MW of net electrical power, as stated in the EU Roadmap to the realisation of fusion energy [3]. As the design of the EU-DEMO is currently in the so-called pre-conceptual design analysis phase [4], the values of its design parameters are still under investigation and might change in the future as technology concepts and accompanying models are updated, and new baselines are released. For this work a EU-DEMO reference reactor is used in order not to relate the analysis to any particular provisional design point. Its relevant engineering parameters, which will be used throughout this paper, are summarised in Table 1.

\begin{tabular}{|l|l|l|l|}
\hline Quantity & Values & Unit & Description \\
\hline$R$ & 9 & $\mathrm{~m}$ & Major radius \\
\hline$B_{T}$ & 6 & $\mathrm{~T}$ & Toroidal magnetic field \\
\hline$q_{95}$ & 3.5 & & Safety factor \\
\hline$\varepsilon$ & 0.31 & & Inverse aspect ratio \\
\hline$f_{L H}$ & 1.2 & & Ratio $P_{\text {sep }} / P_{L H}$ \\
\hline$P_{f u s}$ & 2000 & $\mathrm{MW}$ & Fusion power \\
\hline$P_{L H}$ & 110 & $\mathrm{MW}$ & LH threshold power \\
\hline$\left(B_{p . t a r} / B_{t, t a r}\right)$ & 0.11 & & $\begin{array}{l}\text { Ratio poloidal to } \\
\text { toroidal field at the } \\
\text { target }\end{array}$ \\
\hline$\left(B_{p, \text { omp }} / B_{t, \text { omp }}\right)$ & 0.36 & & Ratio poloidal to \\
\hline
\end{tabular}




\begin{tabular}{|l|l|l|l|}
\hline & & & $\begin{array}{l}\text { toroidal field at the } \\
\text { outer midplane }\end{array}$ \\
\hline$\frac{P_{\text {sep } B}}{q_{95} A R}$ & 8.11 & $\mathrm{MW} \mathrm{T} \mathrm{m}^{-1}$ & $\begin{array}{l}\text { Figure of merit - } \\
\text { divertor reattachment }\end{array}$ \\
\hline$\hat{C}_{Z, \text { det }}$ & 0.85 & & $\begin{array}{l}\text { Figure of merit - } \\
\text { impurity concentration } \\
\text { for detachment }\end{array}$ \\
\hline$\kappa_{95}$ & 1.65 & & Elongation \\
\hline$\delta_{95}$ & 0.33 & & Triangularity \\
\hline
\end{tabular}

Table 1. Relevant parameters of the chosen EU-DEMO reference reactor. A more detailed description of the various quantities can be found in the main text.

The reference plasma scenario for EU-DEMO, as well as for ITER, is the so-called ELMy-H mode [5], which is known to exhibit a threshold on the charged particle power crossing the last closed magnetic surface, $P_{s e p}$. Below this threshold the confinement capability of the machine is significantly reduced as the L-mode is recovered. Different empirical scalings for the threshold power, $P_{L H}$, exist and the most commonly used is the Martin scaling [6].

$$
P_{L H}=0.049 n^{0.72} B_{T}^{0.8} \Sigma^{0.94}
$$

This scaling will be used in the work presented here. For equation $1, n$ is the (line averaged) plasma density expressed in $10^{20} \mathrm{~m}^{-3}$ units, $\Sigma$ is the plasma surface area and $P_{L H}$ is expressed in MW. Most available scaling laws for the LH threshold power $[6,7,8]$ exhibit a positive dependence both on the toroidal magnetic field and on the major radius, via the plasma surface $\Sigma$. Since the power crossing the separatrix is mainly directed onto the divertor plates, it is legitimate to expect the problem of the divertor compatibility to become more severe when the "size" of the reactor (here intended both in terms of radius and of magnetic field) increases. This is true independent of the divertor configuration assumed. The EU-DEMO reactor is assumed to operate with an at least partially, but most probably fully detached divertor, as the power striking on the plates would otherwise be too high to be dealt with by current state-of-the-art high heat flux technology, at least in the conventional lower single null configuration $[9,10]$. In order to achieve the detached state, a significant fraction of $P_{\text {sep }}$ must be dissipated in the scrape-off layer (SOL) before actually reaching the target plate. This high dissipation is intended to be achieved in EU-DEMO with the use of seeded, radiative impurities, such as $\mathrm{Ar}$ or $\mathrm{Kr}$ (together with a core radiator as for example Xe) [9], which redistribute a significant part of the exhaust power onto the first wall in the form of electromagnetic radiation. The use of these impurities is not without consequences for the machine operation. A certain fraction of the seeded atoms are not expected to remain confined in the divertor volume, or in the SOL more generally, but to migrate into the plasma core. There, depending on the edge profile characteristics, these impurities can cause either a reduction of the fusion power via fuel dilution or trigger some radiative instability [11]. For this reason, a plasma configuration which requires a lower impurity concentration to achieve a robust divertor detachment is in general preferable. Also, the high content of impurities required for the steady-state operation makes the addition of further impurities in emergency cases very critical for the stability of the discharge. A figure of merit to make this concept more quantitative, thus allowing a comparison of different reactor designs with regard to this particular aspect, is discussed in this paper.

Furthermore, divertor detachment is a condition that can be lost. Examples may include: as a consequence of a failure of the impurity injection system; or after a large fluctuation in the separatrix power; or for a density fluctuation in the divertor region, or for one or more pellet injectors seizing up. For machines like EU-DEMO, which will possess very stringent safety and availability requirements, it is essential to demonstrate that a strategy to safely terminate the plasma discharge is available, which must not compromise the integrity of the machine in case of accidental reattachment.

The mitigation method must depend on how large the expected heat flux on the target plate in case of divertor reattachment is. The magnitude of the heat flux by reattachment determines in fact the time available to restore detachment, or to terminate the discharge, before the machine suffers severe damage.

In this analysis, both the role of the ELMs and major off-normal events such as disruptions are neglected - they represent of course a serious threaten to the DEMO operation, but this is not the subject of this work. Nevertheless, scaling laws referring to ELMy H-mode are adopted, assuming they represent a valid approximation for the conclusions drawn here. Instead, the analysis is concentrated on the necessary criteria which have to be considered in the preliminary design phase of EU-DEMO to ensure divertor protection, and the impact of those criteria on the machine size. This investigation is intended to provide a conceptual framework for the early phases of the dimensioning and does not purport to enter into the physics and engineering detail of the divertor design. Also, it is important to stress that this work refers solely to a lower single null conventional divertor 
using actively cooled solid metal plasma-facing components and with extrinsic seeding for heat flux dissipation, which is the solution currently adopted for EU-DEMO.

The paper is structured as follows: in section 2, the criteria which have to be considered for the divertor protection are discussed, and suitable figures of merit to compare different possible EU-DEMO configurations are identified from the published literature. In section 3, the dependence of such figures of merit on the relevant design parameters of the machine, namely $R$ and $B_{T}$, is exploited by employing a simple 0D physics model. In section 4 , a broader parameter scan is performed by means of the systems code PROCESS [12,13]. Finally, in section 5, it is discussed to what extent the results obtained for EU-DEMO are applicable to other reactor concepts. Conclusions are drawn in section 6.

\section{Criteria and figures of merit for the divertor protection in EU-DEMO}

As discussed in the introduction, in order to ensure a safe operation for the divertor in EU-DEMO, the two following conditions are required to be fulfilled:

First Criterion: The machine must be able to survive in case of divertor reattachment for a time interval which is sufficiently long to allow a safe, controlled termination of the plasma discharge, or a recovery of detachment.

It is important here to stress that the first wall in a machine like the EU-DEMO is much more fragile than in ITER because of the need to breed tritium. There is only a $\sim 3 \mathrm{~mm}$ metal layer between the plasma chamber and the high pressure coolant [14,15]. Additionally, even perfectly mitigated disruptions in EU-DEMO would lead to a long interruption of the machine operation, with repercussions on its availability - which is an important aspect of the EU-DEMO mission [3]. Thus, the allowable number of disruptions during the EU-DEMO lifetime is much lower than can be tolerated in ITER. For this reason, any emergency plasma shut-down procedure which foresees a plasma/wall contact at high current is undesirable, and is not at present considered viable, as the consequences might be too severe. This implies that there must exist for EU-DEMO a strategy which allows for dealing with divertor reattachment without losing plasma control. More in detail, investigations carried out inside the PPP\&T department have shown, for the current EU-DEMO configuration, that:

- In order to avoid consequences to the first wall, no plasma/wall contact with a plasma current $I_{p}$ larger than $\sim 5 \mathrm{MA}$ can take place [16], whereas $I_{p} \approx 20 \mathrm{MA}$ during the flattop phase. This is a consequence of the mentioned fragility of the EU-DEMO first wall.

- The plasma current cannot be ramped down faster than $0.5 \mathrm{MA} / \mathrm{sec}$ (under very optimistic assumptions with $0.2 \mathrm{MA} / \mathrm{sec}$ being a more reasonable value) without losing the plasma magnetic control and thus having a plasma/wall contact $[17,18]$.

These results lead to the conclusion that every fast shut-down scenario which is viable for EU-DEMO has to include a strategy to protect all PFCs for a sufficient time to ramp down the current to a level where a loss of plasma control becomes manageable (i.e. 30 sec). In spite of its apparent difficulty, this approach looks at the moment preferable to, say, an order-unity variation in the seeded impurities concentration in order to recover detachment. Reason for that is that EU-DEMO is prone to radiative instabilities, i.e. in presence of downwards fluctuations of the heating power, the line radiation from the seeded impurities in the core (Xe) can increase substantially, leading to a further deterioration of the confinement and thus initiating an avalanche-like mechanism. An order-unity relative variation of the Ar concentration would increase the risk for such instability to take place, not only because Ar itself can increase the radiation from the pedestal region as well, but also - and especially - because it degrades the fusion power generation via fuel dilution, thus reducing significantly the plasma heating (recall that alpha particles are by far the dominant plasma heating source in EU-DEMO). Also, the residence time of Ar particles in the plasma chamber is quite long, thus, once injected, it would be quite difficult to remove the excess Ar by means of the available actuators (pumps), hampering the controllability of the recovered discharge.

As shown below, the divertor is not able to survive for such a long time in presence of reattachment in absence of active countermeasures. The definition of a strategy to ensure the integrity of the target plate in the case of loss of detachment avoiding at the same time an early plasma/wall contact is therefore a necessary requirement for every machine with a fragile breeding wall, which has to be taken into account in the preliminary design phases, as these requirements can drive machine dimensioning and hence impact other systems. It is however necessary to stress that divertor reattachment is not acknowledged to be among the most important causes of disruptive events in EU-DEMO, and thus a solution for the reattachment problem is by no means a solution for the disruption avoidance as a whole. Still, the required disruption rate for EU-DEMO is very low, and therefore a careful analysis of all possible initiating events has to be undertaken. 
The target protection strategy depends on how high the heat flux on the plate will be in the case of a loss of detachment. Thus, this criterion naturally translates - at least in this preliminary phase - into a limit on the maximum tolerable heat flux in attached operation.

In this work, it is assumed that an effective countermeasure - namely the divertor sweeping, as discussed in detail below - exists and is able to ensure the integrity of the divertor for the required time lapse up to a certain heat flux. To conclude that divertor sweeping is really a viable solution for EU-DEMO, however, other investigations have to be carried out - e.g. the W influx by reattachment might be anyway too high and cause a disruption. Also, there is the possibility that some technological solution, like the CPS lithium systems [19], could allow to withstand transiently very high heat fluxes in absence of active countermeasures, or some divertor configurations extremely resilient against reattachment, as e.g. Liquid Metal (LM) divertors, might be proven to be operable at reactor scale, but this has not been analysed in detail yet.

The reattachment of the divertor is an unlikely event, but it cannot be excluded, partially due to the limited diagnostic options available which are expected to be compatible with operation in a high neutron fluence environment like the EU-DEMO, and in power plants in general. Additionally, it is of paramount importance to develop for EU-DEMO a diagnostic able to detect a loss of detachment before a complete reattachment occurs. Currently, the feasibility of spectroscopic measurements able to detect the movement of the radiation front along the near SOL field lines is being investigated - as discussed in [18]. The dynamics of divertor reattachment is however not the subject of the present work, which considers the worst case, namely that the plasma is in a state where the transition to reattachment cannot be avoided and countermeasures have to be undertaken.

Second Criterion: the divertor detachment must be achievable with an impurity content which is sufficiently low to avoid the risk of radiative instabilities in the plasma edge, or to avoid an excessive fuel dilution.

As mentioned, the number of disruptions in the EU-DEMO should be minimised. Thus, it is extremely important to reduce the probability of all possible initiating events, such as the onset of a radiative instability. Also, it is known that an excessively large $Z_{\text {eff }}$ in the core can massively deteriorate the fusion power level (and affect the pulse length as well via flux consumption), thus becoming an obstacle for the realisation of the EU-DEMO mission. The effect of the impurities for the divertor detachment on the reactor performance has been extensively analysed in [11].

In the following subsections, these criteria will be expressed in terms of quantitative figures of merit taken from the existing literature.

\subsection{Figure of merit - heat flux by re-attachment}

As discussed, EU-DEMO must be able to withstand the attached heat flux without reaching coolant burn-out conditions for a sufficiently long time in case a loss of detachment - no matter the cause - occurs. In view of the fragility of the EU-DEMO first wall, a discharge termination via an increase in matter injection, which can highly enhance the risk of a disruption even after the recovery of the detachment, is not considered as a viable solution to deal with reattachment. Thus, alternative approaches have to be explored. The parallel heat flux at the divertor plate can be calculated as [20]

$$
q_{\text {tar }}=f_{\text {mag }} \frac{P_{\text {tar }}}{2 \pi R \lambda_{\text {int }}} \sin (\eta)
$$

Where $\lambda_{\text {int }}$ is the power decay length for the heat load at the divertor plate, and the coefficient $f_{\text {mag }}$

$$
f_{\text {mag }}=\frac{B_{p, t a r}}{B_{p, o m p}}
$$

takes into account the different pitch of the magnetic field line between the outer midplane (subscript $o m p)$ and the outer target (subscript tar). According to the values in Table 1 for the reference EU-DEMO the value $f_{\text {mag }}=0.29$ is found (these values originate from a magnetic equilibrium calculation for the EU-DEMO baseline 2017 performed with the code CREATE [21], and assumed still valid for the considered EU-DEMO reference). In turn, the angle $\eta$ is introduced as the angle between the striking magnetic field line and the divertor plate in the poloidal plane. For the current - and preliminary - EU-DEMO design, its value is $\eta=30^{\circ}$ [22].

The decay length on the target plate $\lambda_{\text {int }}$ is related to the decay length at the outer midplane $\lambda_{q}$ via the relation [23]

$$
\lambda_{\text {int }}=\lambda_{q}+1.64 S
$$


It is expected that the parameter $S$, which mimics the broadening of the heat channel in the divertor volume by effect of perpendicular transport processes, will be larger in devices like EU-DEMO than in existing machines, mainly by virtue of a larger divertor volume - this assumption has been often adopted in the EU-DEMO literature, see e.g. [24]. It is for this reason that the value of $S$ is assumed to be:

$$
S=\lambda_{q}
$$

which is larger than what normally observed in the existing experiments, where $S \approx 0.5 \lambda_{q}$. The consequences of adopting $S=0.5 \lambda_{q}$ will be anyway discussed in the following. The power decay length $\lambda_{q}$, which is proportional to $\lambda_{\text {int }}$ according to Eq.4 and 5, is assumed in the present work to scale as suggested by the Eich scaling $[25,26]$.

$$
\lambda_{q}=0.73 B_{T}^{-0.78} q_{95}^{1.2} R^{0.1}
$$

where $q_{95}$ is the value of the safety factor on the magnetic surface which encloses the $95 \%$ of the magnetic flux of the confined plasma. Recent work [27] suggests that, under ITER and EU-DEMO relevant conditions, turbulence will be able to broaden the heat flux channel, possibly significantly. Furthermore, a highly radiative SOL is expected to lead to an increase in $\lambda_{q}$ [28], so the use of the Eich scaling could lead to overly conservative conclusions. In this work, however, the Eich scaling is still used, following the published literature on the EU-DEMO scenario development $[9,10]$, whereas some margin is left on the choice of $S$. Eq. 6 introduces an (almost) inverse proportionality between the power decay length $\lambda_{q}$ and the poloidal magnetic field $B_{p}$ (entering via the safety factor).

$$
\lambda_{q}=0.73 B_{T}^{0.42}\left(\frac{\varepsilon}{B_{p}}\right)^{1.2} R^{0.1} \approx 0.73 \frac{\varepsilon}{B_{p}}
$$

having written

$$
q_{95}=\varepsilon \frac{B_{T}}{B_{p}} .
$$

The somewhat crude approximations adopted here are justified by the fact that a figure of merit - which is the goal of the present derivation - is not intended to be quantitatively accurate, but rather it has to be able to catch the main dependencies of a complex physical phenomenon on some relevant engineering parameters.

In view of Eq.2 and 7, the heat flux by re-attachment $q_{\text {tar }}$ - at constant $f_{\text {mag }}$ and $\eta$, which can be assumed to be optimised values for the EU-DEMO lower single null configuration - can be shown to be proportional to

$$
q_{t a r} \propto \frac{P_{t a r} B_{p}}{R} \propto \frac{P_{t a r} B_{T}}{q_{95} A R}
$$

where $A=1 / \varepsilon$. A heat flux limit on the target can be thus expressed via an upper limit on this quantity, namely

$$
\frac{P_{s e p} B_{T}}{q_{95} A R}<\left.\frac{P_{s e p} B_{T}}{q_{95} A R}\right|_{R E F}
$$

as already suggested in [29]. The constant $P_{s e p} B_{T} /\left.q_{95} A R\right|_{R E F}$ is here introduced, and it is related to the maximum tolerable heat flux that EU-DEMO can withstand from a technological point of view. The determination of its magnitude is discussed in detail in the next subsection. Note that Eq.10 refers to $P_{\text {sep }}$ instead of $P_{\text {tar }}$, the latter being unsuitable for usage as a figure of merit based on engineering parameters. The two quantities are linked, especially in attached divertor conditions, when dissipation does not dominate. This is discussed in more detail below.

It is worth highlighting once more that the value of $P_{s e p} B_{T} /\left.q_{95} A R\right|_{R E F}$ should not be taken as a physical constant with universal significance. Rather, it represents a quantity which is linked to the technological solution adopted. For example, divertor solutions other than the conventional ITER-like lower single null adopted for the EU-DEMO might allow the achievement of higher values of $P_{s e p} B_{T} /\left.q_{95} A R\right|_{R E F}$. This aspect is discussed in more detail in section 5 . In particular, in the case that a divertor configuration could be shown to not undergo reattachment in any circumstance (e.g. LM), the entire argument will no longer be valid. 
Also, the calculation below should not be understood as a design choice justification, as for this purpose more substantiated analyses would be required.

\subsection{Heat Load by Re-attachment and Divertor Sweeping}

To calculate the heat load on the target plate when the divertor is attached, the simplified approach shown in [20] is adopted. First, one has to determine the wetted area $A_{W}$ on which the heat is deposited.

$$
A_{w}=\frac{P_{\text {tar }}}{q_{\text {tar }}}
$$

Substituting Eq.2 in Eq.11, one finds

$$
A_{w}=\frac{2 \pi R \lambda_{i n t}}{f_{m a g} \sin (\eta)}
$$

Following the ITER Heat Load Specification [30], it is assumed that $2 / 3$ of the power crossing the separatrix is directed towards the outer target, and that a fraction of that power, $f_{\text {loss }}=20 \%$, is dissipated in the SOL by various processes even in the absence of detachment (e.g. charge exchange, hydrogen radiation, residual impurity radiation). Thus, the value of the power striking the divertor plate by reattachment, $P_{t a r}$, can be related to $P_{s e p}$ via:

$$
P_{\text {tar }}=\frac{2}{3}\left(1-f_{\text {loss }}\right) P_{\text {sep }}
$$

The EU-DEMO divertor is based on water cooled monoblocks with a $\mathrm{W}$ coating with cooling pipes made of $\mathrm{CuCrZr}$ and able to tolerate a stationary heat flux up to $20 \mathrm{MW} \mathrm{m}^{-2}$ [22]. Simulations performed with the code RACLETTE [31] and data shown in Fig. 1 indicate that the use of divertor sweeping [32] can allow the EU-DEMO target to survive for a longer time (tens of seconds) for power densities up to $\sim 70 \mathrm{MW} / \mathrm{m}^{2}$. In the calculations shown in Fig.1, three criteria have been considered for the EU-DEMO to prevent monoblock failure, namely that the:

- Temperature of tungsten armour is lower than the tungsten recrystallization temperature $1200{ }^{\circ} \mathrm{C}-$ this is to some extent a simplification, as in fact other parameters such like the depth of the recrystallization and the number of stress cycle can influence the behaviour or the target, but a precise treatment of these phenomena goes beyond the purposes of the present analysis.

- Temperature on the $\mathrm{CuCrZr}$ pipe is lower than $350^{\circ} \mathrm{C}$ (softening temperature)

- Heat flux to the coolant is lower than the critical heat flux (CHF)

If no active control is employed at $70 \mathrm{MW} / \mathrm{m}^{2}$, burn-out in the coolant is reached in less than two seconds. This time interval is too short for any non-disruptive plasma termination strategy, being also shorter than the expected energy confinement time of the core plasma. On the contrary, during the sweeping phases, the strike points on the divertor plate are moved periodically in the poloidal direction, with a set amplitude and frequency. This allows spreading of the power over a larger surface than in the absence of sweeping. Detailed EM calculations illustrated in [32] show that a frequency of $1 \mathrm{~Hz}$ is obtainable in EU-DEMO without excessive AC losses on the required timescales (i.e. below 1 minute). Higher frequencies are more challenging from a technological point of view. Thermal fatigue prevents the employment of divertor sweeping during the normal flat-top plasma operation. The possibility of using divertor sweeping in EU-DEMO is at this stage still speculative, as there are many open points to be addressed, both concerning the physics and engineering aspects. Also, from an operative point of view, it is crucial to designate diagnostics able to ensure that the sweeping can already be active when the plasma reattaches - as for example the spectroscopic detection of the radiative front previously mentioned. 


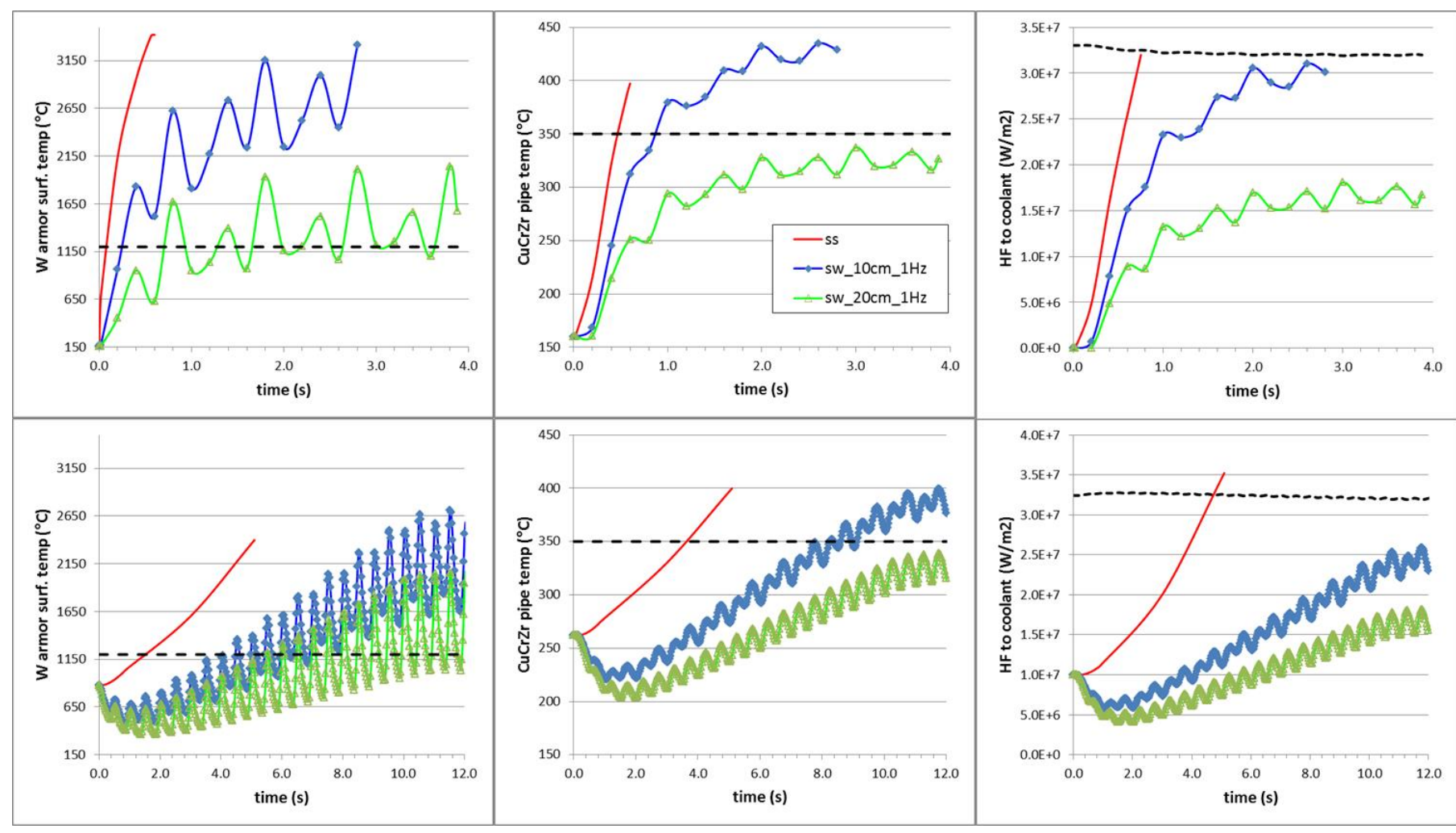

Figure 1. RACLETTE simulation using as input $70 \mathrm{MW} / \mathrm{m}^{2}$ heat flux. In the first row, a step variation in the heat flux from 10 to $70 \mathrm{MW} / \mathrm{m}^{2}$ is assumed, whereas the second row refers to a $10 \mathrm{~s}$ ramp from 10 to $70 \mathrm{MW} / \mathrm{m}^{2}$. The curves refer to steady state (red), sweeping at $1 \mathrm{~Hz}$ of $+/-5 \mathrm{~cm}$ (i.e. $10 \mathrm{~cm}$ peak to peak - blue) and $+/-10 \mathrm{~cm}$ (i.e. $20 \mathrm{~cm}$ peak to peak - green), with considered limits represented as black dashed line. For both rows, from left to right, the following quantities are plotted: i) Temperature on tungsten armour (recrystallization limit $=1200{ }^{\circ} \mathrm{C}$ ). ii) Temperature on $\mathrm{CuCrZr}$ pipe (softening limit $=350{ }^{\circ} \mathrm{C}$ ). iii) $\mathrm{Heat}$ flux to coolant, with variable Critical Heat Flux limit. Note the different time durations of the calculations between first and second row.

The effect of the divertor sweeping can be shown to be extremely beneficial. Fig. 1 shows that with a spatial extent of $\pm 10 \mathrm{~cm}$ (i.e. $20 \mathrm{~cm}$ peak to peak) around the unperturbed strike point (blue curves) the critical heat flux is never reached. However, the temperature of the tungsten armour oscillates around the recrystallization temperature. The recrystallization of the tungsten is not an immediately dangerous process, unlike burn-out and softening of $\mathrm{CuCrZr}$, which can lead to instantaneous damage. Thus, the value of $70 \mathrm{MW} / \mathrm{m}^{2}$ is identified as a good estimate for the technological heat flux limit EU-DEMO is able to deal with at the current design stage. Taking into account a $15 \%$ safety margin, a value of $q_{\text {tar,max }}=60 \mathrm{MW} / \mathrm{m}^{2}$, where $q_{\text {tar,max }}$ is the maximum heat flux by reattachment at which the integrity of the divertor can be ensured in presence of divertor sweeping for a time compatible with the plasma current ramp-down, is adopted in the rest of the presented analysis.

The behaviour of a divertor monoblock both in the presence of a stepwise change in $q_{t a r}$ (from 10 to $70 \mathrm{MW} / \mathrm{m}^{2}-$ first row) and in the presence of a $10 \mathrm{sec}$ ramp between the same values have been considered in Fig.1. The first approach is typically used to analyse the response of a system, whereas the second one is more closely aligned to a real world scenario, as the loss of detachment, especially in a large device, is not sudden but rather quite slow. Nevertheless, the two cases show a similar behaviour once the maximum heat flux is reached. This is due to the ability of the divertor monoblocks to reach a thermal equilibrium state in a short time. Also, it is interesting to note, for the second case, that in absence of sweeping the CHF is reached before the maximum $q_{\text {tar }}$.

By employing the Eich scaling, Eq.7, as well as Eq.4 and 5, and using the EU-DEMO reference parameters in Table 1 , one finds $\lambda_{q}=1.32 \mathrm{~mm}$ and $\lambda_{\text {int }}=3.49 \mathrm{~mm}$. With $\eta=30^{\circ}$ and $f_{\text {mag }}=0.29$, the wetted area, $A_{w}$, amounts to $1.35 \mathrm{~m}^{2}$. Taking the maximum allowable heat flux, $q_{\text {tar,max }}=60 \mathrm{MW} / \mathrm{m}^{2}$, and using Eq. 11,12 and 13 , this corresponds to a power at the separatrix, $P_{s e p}$, of $150 \mathrm{MW}$. Equivalently it can be given as $f_{L H}=1.36$, where $f_{L H}$ is the ratio between $P_{s e p}$ and $P_{L H}$, the value of the latter being also reported in Table 1. In terms of the figure of merit $P_{\text {sep }} B_{T} / q_{95} A R$, Eq.10 translates finally into

$$
\frac{P_{s e p} B_{T}}{q_{95} A R}<\sim 9 M \frac{T}{m}
$$

This reference value will be adopted in the presented work as a limiting value for EU-DEMO concerning the divertor reattachment figure of merit. At $f_{L H}=1.2$, which is the value the reference EU-DEMO is supposed to be operated at (see Table 1), one finds $P_{\text {sep }}=130 \mathrm{MW}$ and $P_{\text {sep }} B_{T} / q_{95} A R=8.11 \mathrm{MW} \mathrm{T} / \mathrm{m}$. It has however to be stressed that, if 
the very conservative assumption $S=0.5 \lambda_{q}$ had been made, then the currently assumed sweeping performance would not have been sufficient to prevent the damage of the divertor at the currently foreseen $P_{\text {sep }}$, and other solutions had to be investigated - e.g. increasing the sweeping frequency allowing for larger AC losses. The work presented in this paper is however not meant to be a justification of the current design - as previously stated.

Instead, the crucial point is that there must exist a solution to protect the divertor for a sufficiently long time during the emergency current ramp down, and this solution introduces a design constraint on the maximum allowable heat flux by reattachment.

\subsection{Figure of merit - critical impurity concentration}

After discussing the maximum tolerable heat flux by reattachment, an analysis of the second figure of merit is presented here. EU-DEMO is assumed to be run with a (partially or, more probably, fully) detached divertor. The detached condition is achieved, and maintained, via injection of seeded radiating impurities. The impurity content in the SOL cannot be arbitrarily high. In fact, if the concentration in the confined plasma region (which is expected to be related to the concentration in the $\mathrm{SOL}$ ) exceeds some critical value, the plasma performance will be compromised. Depending on the radiative properties of the seeded impurity species, and also on the plasma kinetic profiles, the plasma performance deteriorates either in terms of stability because of excessive radiation in the pedestal, or via fuel dilution. To determine the link between the impurity concentration in the SOL and in the core plasma is not straightforward, as it depends on impurity transport mechanisms whose extrapolation to reactor relevant scales is not completely clear, and which goes beyond the purposes of this manuscript. In current experiments, a compression factor $c_{F}$

$$
c_{F}=\frac{c_{Z, S O L}}{c_{Z, \text { core }}}
$$

of the order of $\sim 5$ is observable [33], varying somewhat for different impurity species [28] (here, $c_{Z}$ identifies the concentration of the generic impurity labelled with $Z$ ).

In a recent publication by Reinke [34], a simple OD argument has been suggested to estimate the necessary concentration of impurities, $c_{Z, \text { det }}$, to obtain divertor detachment for a given tokamak machine, with known engineering parameters. Neglecting some factors which will be kept constant in this analysis, the Reinke formula (Eq.10 in Reinke [34]) reads

$$
c_{Z, d e t} \propto f_{L H}^{1.14} B_{T}^{0.88} R^{1.33}
$$

Here, the derivation of the formula is not repeated, and the interested reader is referred to the cited paper. This criterion is based on the Martin scaling for the evaluation of $P_{s e p}=f_{L H} P_{L H}$, and also on the Eich scaling for the heat flux channel width. In this sense, the two figures of merit used in this paper are fully compatible; a change in choice of LH scaling or SOL scaling would thus require the adjustment of both figures of merit. Although the Reinke scaling is too oversimplified to be used for a quantitative evaluation of the necessary impurity concentration, it represents a useful tool for comparing different machines and/or configurations. A similar criterion has been proposed by Goldston et al. [35], where the power $P_{\text {sep }}$ was left as a free parameter and not expressed in terms of $P_{L H}$ as in [34]. In this work, $P_{S e p}$ is conveniently related to $P_{L H}$, since maintaining the $\mathrm{H}$-mode is one of the requirements for the EU-DEMO operation, so the approach of [34] is adopted. However, it is possible to demonstrate that, employing the criterion Eq.9 in [35], Eq.16 would then be written as

$$
c_{Z, d e t} \propto f_{L H} B_{T}^{0.52} R^{1.16}
$$

(having used Eq.22 below and assuming $P_{s e p}=f_{L H} P_{L H}$, calculating the latter with the Martin scaling). For the purposes of this work, which are quantitative but to some extent speculative, the two criteria can be considered equivalent. It is however interesting to note that the two scaling laws exhibit a rather different dependence on the tokamak aspect ratio, which originates from the different expression chosen for $\lambda_{q}$. Here, no attempt is made to indicate which of the two approaches better captures the experimentally observable dependence of $\lambda_{q}$ on $A$. For consistency however, the Reinke criterion, which is based on the Eich scaling like the $P_{s e p} B_{T} / q_{95} A R$ figure of merit, is employed in this manuscript. The figure of merit for the critical impurity concentration can then be expressed, in a manner analogous to the previous case, as

$$
c_{Z, \text { det }}<c_{Z, R E F}
$$

where $c_{Z, R E F}$ is again a reference value which has to be defined to guarantee the machine operation within some safety margin. Plasma simulations show that a machine like the reference EU-DEMO is still operable, marginally, with the required impurity concentration to detach the divertor, as discussed in [36], where fluid SOLPS simulations 
with $\lambda_{q}=3 \mathrm{~mm}$ were carried out. Clearly, a more detailed assessment would be required at this stage to determine the correct value of $c_{Z, R E F}$, but for the purpose of this simplified analysis a rough estimate is employed. In particular, with some degree of arbitrariness, it is assumed that $c_{Z, R E F}$ is equal to the EU-DEMO value of $c_{Z, \text { det }}$ if $P_{\text {sep }}=150$ $\mathrm{MW}$ - which corresponds to $f_{L H}=1.36$, as already stated in the previous subsection. In other words, it is assumed that at $f_{L H}=1.36$, the reference EU-DEMO would be operated at the maximum tolerable value for both figures of merit at the same time. Eq.18 can therefore be recast as

$$
\hat{c}_{Z, \text { det }}<1
$$

where the hat ${ }^{-}$indicates quantities normalised to the mentioned reference EU-DEMO at $f_{L H}=1.36$. Strictly speaking, if a reactor configuration is operated at $\hat{c}_{Z, \text { det }}>1$, it possesses an impurity concentration in the SOL (and thus in the core, assuming identical compression factors) larger than what the reference EU-DEMO would require to maintain the detachment at $P_{s e p}=150 \mathrm{MW}$. In reality, this occurrence is not necessarily implying that the stability of the scenario or its fusion yield are compromised - more careful analysis would be required at that point. However, it is reasonable to assume that the reference EU-DEMO at $f_{L H}=1.2$ operates close to the maximum tolerable impurity concentration, but still has some margin. This choice has been at least approximately justified by the simulations presented in [11], but it is however not the most conservative case - as the mentioned SOLPS simulations assume a broader $\lambda_{q}$ than predicted by the Eich scaling. In view of Eq.16, the reference EUDEMO at $f_{L H}=1.2$ operates at $\hat{c}_{Z, d e t}=0.85$, as stated in Table 1 .

\section{Allowable machine size for EU-DEMO}

The goal of the following calculations is to express the two relevant figures of merit for the divertor protection shown above - namely $P_{\text {sep }} B_{T} / q_{95} A R$, and $\hat{c}_{Z \text {,det }}$ - as a function of $f_{L H}$, the major radius $R$ and the magnetic field $B_{T}$, the latter two being, possibly, the most important engineering parameters in a preliminary reactor dimensioning. The analysis will be carried out with the purpose of identifying the configurations which are not feasible in terms of divertor protection for the EU-DEMO.

The following quantities are kept constant:

- Fusion power $P_{f u s}$. The actual EU-DEMO design aims to produce $2 \mathrm{GW}$ of fusion power, which, according to PROCESS simulations, allow an electricity production of $500 \mathrm{MW}$ - see e.g. [18]. The assumption of constant fusion power is therefore to be understood as a proxy for constant electricity production, which is a reasonable criterion to compare different reactor configurations (it is assumed that the recirculating power does not vary significantly with $R$ and $B_{T}$ if $P_{f u s}$ is kept constant).

- Edge Safety factor $q_{95}$. It is assumed that the value of $q_{95}$ is always chosen to be the minimum which allows a sufficient stability margin against disruptive events.

- Greenwald fraction $f_{G W}$. Again, the machine is supposed to be operated at the maximum density value compatible with the density limit, to maximise the performance at a given $R$ and $B_{T}$.

The design space where the constraints on the two figures of merit are fulfilled is identified by means of a simple OD argument, keeping shape parameters like aspect ratio and elongation constant, in order to focus on the $R$ and $B_{T}$ dependency. In section 4, a broader parameter space has been explored using the systems code PROCESS.

\subsection{OD Model}

The definition $P_{\text {sep }}=f_{L H} P_{L H}$ is used and $P_{L H}$ is given by the Martin scaling [6]. The surface $\Sigma$, with the present assumptions, is proportional to $R^{2}$. In turn, the plasma density $n$ is linked to the plasma current $I_{p}$ via the Greenwald fraction $f_{G W}=\pi n a^{2} / I_{p}$, which has been assumed to be constant,

$$
n=f_{G W} \frac{I_{p}}{\pi a^{2}}
$$

The plasma current $I_{p}$ can be written as a function of $R$ and $B_{T}$ by exploiting the constancy of $q_{95}$ and of the shape factors

$$
I_{p}=f_{q}(\kappa, \delta) \frac{a^{2}}{R q_{95}} B_{T}
$$


where $f_{q}(\kappa, \delta)$ is a function of plasma elongation $\kappa$ and triangularity $\delta$, which for the present analysis is constant. In terms of radius and field

$$
I_{p} \propto R B_{T}
$$

This implies

$$
n \propto \frac{B_{T}}{R}
$$

Substituting Eq.23 in Eq.1 and neglecting constant factors, it can be concluded that

$$
P_{L H} \propto B_{T}^{1.52} R^{1.16}
$$

Following [37], but neglecting the role of impurities, the fusion power is written as

$$
P_{f u s}=c_{1} \beta_{N}^{2} \frac{B_{T}^{4} R^{3}}{q_{95}^{2} A^{4}}
$$

This leads to the proportionality relation for average plasma pressure, $p$ :

$$
p^{2} \propto R^{-3}
$$

which follows from having used the dependence of $I_{p}$ on $R$ and $B_{T}$ derived in Eq.22, and enforcing the constancy of the fusion power $\left(c_{1}\right.$ is a constant whose value is for this analysis not relevant, but can be determined from the condition $P_{f u s}=2 \mathrm{GW}$ for the EU-DEMO parameters in Table 1). Also, as both the safety factor and the aspect ratio are assumed to be constant, the average plasma pressure, which enters via the normalised pressure parameter $\beta_{N}$, can be understood as the value of the (diamagnetic) energy $W$ per unit volume $V$, where the volume is proportional to $R^{3}$ - the shape having been fixed.

$$
V \propto R^{3}
$$

In turn, the energy $W$ can be taken as proportional to $P_{\text {fus }}$ (constant) times the confinement time $\tau_{E}$ (note that these assumptions imply that auxiliary power sources are neglected, i.e. it is assumed that the machine is ignited or at least with a sufficiently high gain, $Q$ ). This leads to

$$
\tau_{E}^{2} \propto R^{3}
$$

Neglecting the influence of the core radiation on the confinement, the standard, non-radiation corrected IPB98(y,2) scaling [4] is used to evaluate the dependence of $\tau_{E}$ on field and radius

$$
\tau_{E} \propto I_{p}^{0.93} B_{T}^{0.15} n^{0.41} R^{1.97} P_{\text {loss }}^{-0.69}
$$

As before, constant terms have been dropped. The power term in the scaling law, $P_{\text {loss }}$, is assumed to be equal to the $\alpha$-particle heating power, $P_{\alpha}$, which is proportional to $P_{f u s}$, thus constant in the present calculation and therefore not considered hereafter. The assumption $P_{\text {fus }} \propto P_{\text {loss }}$ is consistent with the use of IPB98(y,2), where the role of core radiation in determining the confinement time is not considered. It should be mentioned that, in the PROCESS calculations below, the power radiating from the innermost region of the plasma core is subtracted from the loss power, as it is assumed not to play a role in the conducted power losses driving the processes captured by the scaling law (for more details see $[38,39]$ ). The influence of the core radiation on the confinement time is still unclear and to some extent debated in the transport community, see for example the discussion in [40] and the experimental results in [41]. However, for typical EU-DEMO parameters, where the inner core region $\left(\rho_{T}<0.6\right)$ radiation is $\sim 25 \%$ of $P_{\alpha}$, the difference between the two approaches is normally around $10-15 \%$ for $\tau_{E}$.

In view of Eq.22, 23 and 28, the confinement time can then be shown to be proportional to

$$
\tau_{E} \propto R^{2.49} B_{T}^{1.49}
$$

Which, once substituted in Eq.27, leads to

$$
B_{T} \propto R^{-0.66}
$$

Eq.30 relates $B_{T}$ to $R$ under the assumption of constant fusion power. 
Using Eq.24 and 30, and $P_{s e p}=f_{L H} P_{L H}$, the two figures of merit for the divertor protection can be expressed as a function of $f_{L H}$ and $R$ only

$$
\begin{aligned}
& \frac{P_{\text {sep }} B_{T}}{q_{95} A R} \propto f_{L H} R^{-1.5} \\
& \hat{c}_{Z, \text { det }} \propto f_{L H}^{1.14} R^{0.75}
\end{aligned}
$$

Incidentally, if the Goldston scaling would have been employed, Eq.32 would read $\hat{c}_{Z, \text { det }} \propto f_{L H} R^{0.81}$, which is not significantly different than the present form based on Reinke scaling. Eq.31 and 32 can be used to plot curves at constant $P_{S e p} B_{T} / q_{95} A R$ and $\hat{c}_{z}$ in the $f_{L H}-R$ plane and, by using Eq. 30 , in the $f_{L H}-B_{T}$ plane. The two representations are equivalent with regard to the link between $R$ and $B_{T}$ in identifying the regions of the parameter space where EU-DEMO would be able to fulfil both divertor protection criteria. Results are shown in Fig. 2, where the chosen reference EU-DEMO $\left(f_{L H}=1.2\right)$ is depicted as a red point, and the limit curves at constant $\hat{c}_{z, d e t}=1$ and $P_{\text {sep }} B_{T} / q_{95} A R=9 \mathrm{MW} \mathrm{T} / \mathrm{m}$ are represented. Also, a lower limit on $f_{L H}$ has been imposed, as EU-DEMO has to be operated above the LH threshold. As the favourable effect of the heavy impurities on $P_{L H}$ has been neglected, it is thus assumed that $f_{L H}=1$ is still an acceptable working point.

The region of the parameter space which would allow the existence of a 2 GW fusion power EU-DEMO reference are shaded in green in Fig.2, and marked with the letter A. As one can see, most of the points in these regions foresee an operation with a power at the separatrix closer to $P_{L H}$ than what currently planned for EU-DEMO. This could possibly exacerbate the problem of $\mathrm{H}$-mode controllability. Areas marked with the letter $\mathrm{B}$ are also allowing the existence of a $2 \mathrm{GW}$ fusion power EU-DEMO, but for their realisation, a more advanced magnetic technology than the one currently used would be needed to provide a higher magnetic field in a smaller space.

On the contrary, points in the regions $\mathrm{C}, \mathrm{D}, \mathrm{E}$ and $\mathrm{F}$ are not suitable for reactor operation and are detailed below:

1. Points in region $\mathrm{C}$ operate below the $\mathrm{L}-\mathrm{H}$ threshold and thus are not providing the required fusion power due to insufficient confinement. Although acceptable in terms of the two figures of merit, they cannot be considered viable for EU-DEMO.

2. Points in region $D$ are unsuitable with respect to both figures of merit.

3. Points in region $E$ are unsuitable with respect to the Reinke criterion, thus requiring an excessively high impurity concentration to maintain detachment.

4. Points in region $\mathbf{F}$ are unsuitable in terms of $P_{s e p} B_{T} / q_{95} A R$ and thus are unable to deal with loss of detachment with the current heat removal technology.
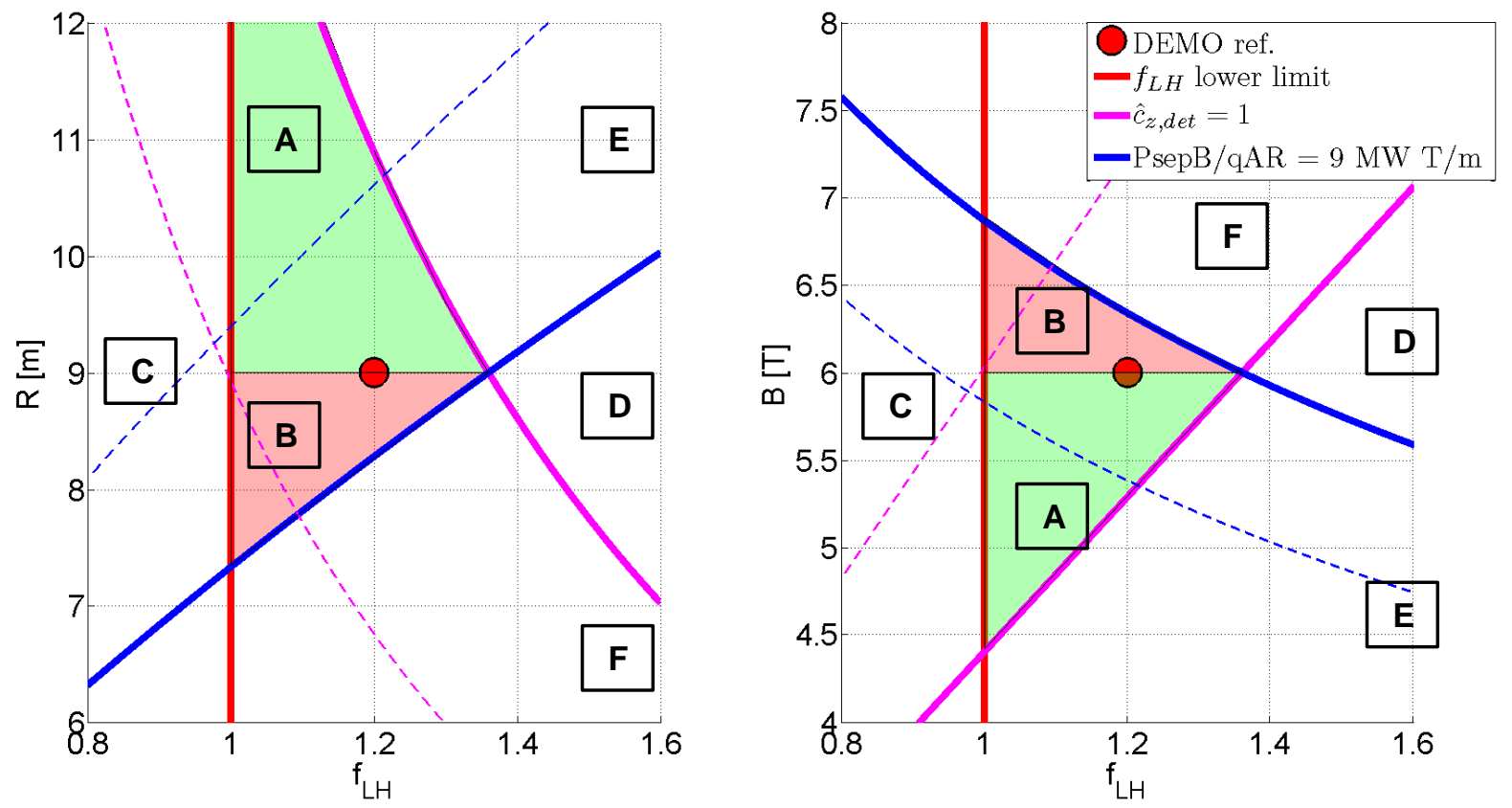

Figure 2. Representation of the constant $\hat{c}_{z, \text { det }}=1$ and $P_{s e p} B_{T} / q_{95} A R=9 \mathrm{MW} \mathrm{T} / \mathrm{m}$ in the $f_{L H}-R$ plane (left) and $f_{L H}-B_{T}$ plane (right), the fusion power $P_{f u s}=2 \mathrm{GW}$ being assumed constant . The design point EU-DEMO reference is represented with a red point. The green shaded areas identify the feasible EU-DEMO configurations, the red shaded areas identify the configuration which would be feasible in presence of an improved, less space-demanding magnet technology. A description of 
regions $\mathbf{A}-\mathbf{F}$ is to be found in the main text. The dashed lines represent the conservative cases $S=0.5 \lambda_{q}$ (blue) and $\widehat{c}_{Z}=\mathbf{0 . 7}$ (magenta). If these more restrictive criteria were selected, no solution for EU-DEMO would exist.

The most relevant fact emerging from Eq.31 and 32 is that the critical impurity concentration $\hat{c}_{Z, d e t}$ scales favourably with $B_{T}$ field and unfavourably with $R$, whereas $P_{s e p} B_{T} / q_{95} A R$ scales favourably with $R$ and unfavourably with $B_{T}$. From a physical point of view, this means that the critical divertor issue for machines at large radius and moderate field is represented by the necessary high concentration of seeded impurity, whereas more compact devices at high field are in general more problematic with respect to divertor reattachment. One could thus state that there exists both an upper and a lower limit for the EU-DEMO major radius, and correspondingly for its magnetic field, beyond which at least one of the two criteria for the divertor protection is violated. Once more, it is stressed that both the value of the maximum allowable impurity concentration and of the maximum allowable heat flux by reattachment are valid only for a conventional divertor with seeded impurities for power dissipation.

Also, it is interesting to note that at $f_{L H}=1.2$ the minimum allowable radial size of EU-DEMO is found to be $R \approx$ $8.3 \mathrm{~m}$. Therefore, unless the machine is operated closer to the LH-threshold - with all the associated risks - the impact on the size of a more compact magnet technology would be limited. Also, these calculations ignore radial build considerations; as the magnetic field on the TF coil increases the coil must get larger to withstand the stresses, and so the achievable field is also limited by the space available for the coil.

Note that the divertor compatibility, although important, is not the only technological criterion to determine the allowable size of a device. For example, small machines at high field have to withstand a higher neutron flux per unit surface, which ultimately shortens the lifetime of the component as discussed in [11]. Purpose of this work is to point out the role of divertor protection in reducing the number of viable options.

Finally, it is interesting (and important) to notice that, if a more conservative approach would be chosen (i.e. $S=0.5 \lambda_{q}$ and $\hat{c}_{Z}=0.7$ as limiting concentration value), no solution would exist for EU-DEMO, as the maximum allowable $P_{\text {sep }}$ should be lower than $\boldsymbol{P}_{L H}$. Again, it is stressed that this paper is not intended to offer a validation of the current EU-DEMO design, which would need more sophisticated tools, but to explore the impact of the divertor protection on the machine dimensioning.

\section{Effect of aspect ratio and shape}

To explore the impact of varying aspect ratio on the operational domain, the PROCESS systems code [12,13] was used. PROCESS contains a self-consistent 0-D plasma model, allowing the effects of a change of aspect ratio on magnetic field and stable elongation to be reflected in the plasma current, energy confinement time as well as in other aspects. PROCESS can allow the components of the radial build to vary in size and position. This includes the magnets, which will be sized such that the resulting machine build satisfies the constraints (e.g. maximum allowed field and pulse length). Concerning divertor protection however, PROCESS - at least in the version employed in this analysis - uses the figures of merit described previously. However, it allows exploring more easily the role of parameters such like elongation and triangularity than the analytical argument developed in the previous sections. Thus, the same considerations on the uncertainty of the value of $P_{\text {sep }} B_{T} / q_{95} A R$ and $\hat{c}_{z}$ discussed in the previous sections apply here as well. The interested reader is referred to $[12,13]$ for a comprehensive description of the assumptions in the code. Plots of the operational space at aspect ratio $A=2.6,3.1,3.6$ (with respective elongations $\kappa_{95}=1.70,1.65,1.56-$ these values originate from an analysis carried out with the method described in [42]) are shown in Fig. 3. For these cases, fusion power was kept constant at $2000 \mathrm{MW}$ as in the EU-DEMO reference assumed in this work, but the pulse length was allowed to vary, its lowest limit having been fixes at $1.33 \mathrm{hrs}$. These plots include the maximum field on the TF coils for reference (dotted lines). It is interesting to note that, due to the complex interactions between variables, the magnetic field contours are rotated with respect to the simple 0-D model described above, although the relationship between the magnetic field and the Reinke and divertor-protection contours is similar. The rotation of the field contours is caused by an increase in $f_{L H}$ corresponding to a decrease of core radiation due to lower impurity levels. This also means less plasma dilution, and so at constant $R$ and fusion power, the magnetic field falls to prevent the fusion power rising. Note also that, for the aspect ratio scan, a more complete version of the Reinke formula has been adopted, namely

$$
c_{Z, \text { det }} \propto f_{L H}^{1.14} B^{0.88} R^{1.33} A^{-0.59}\left(1+\kappa^{2}\right)^{-0.64}
$$



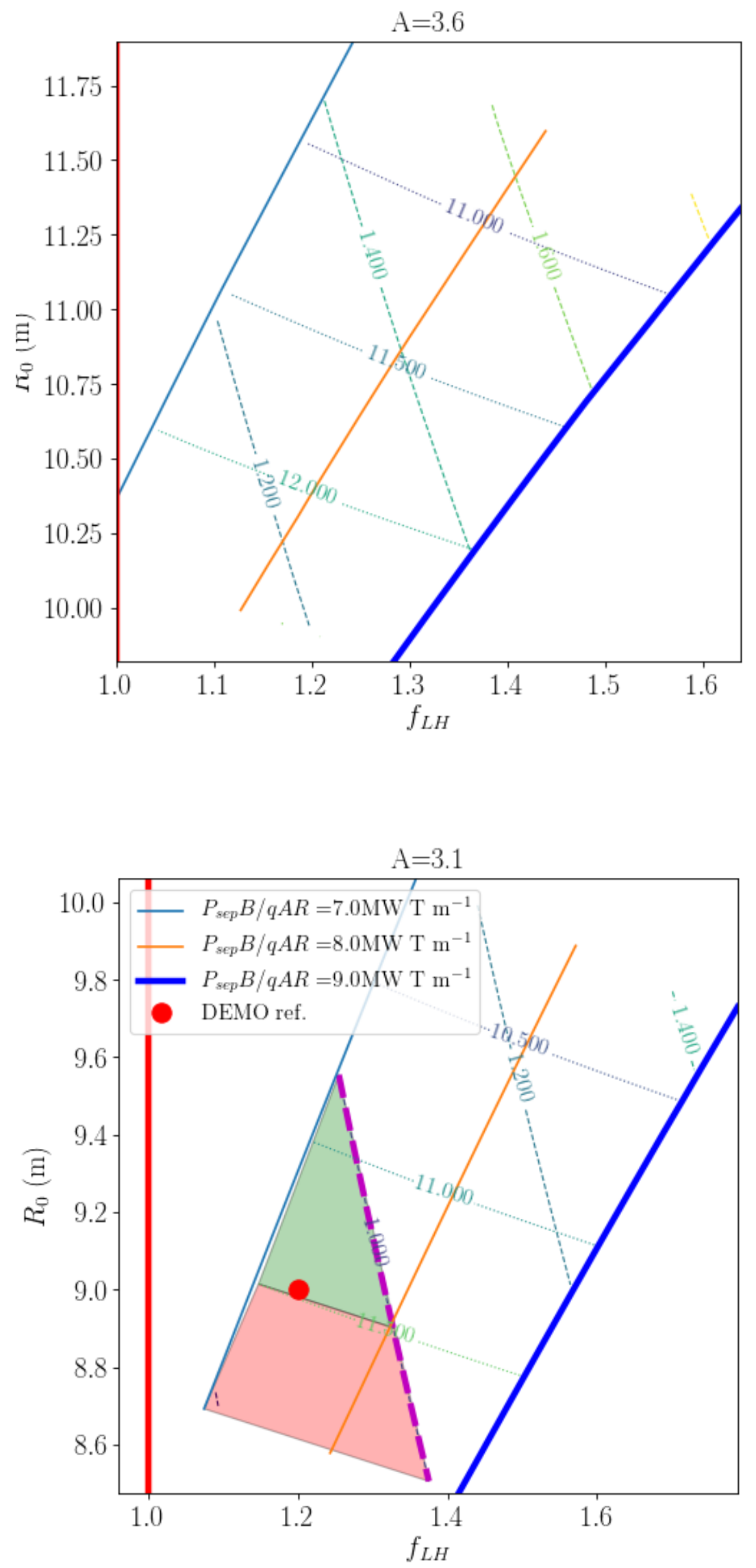


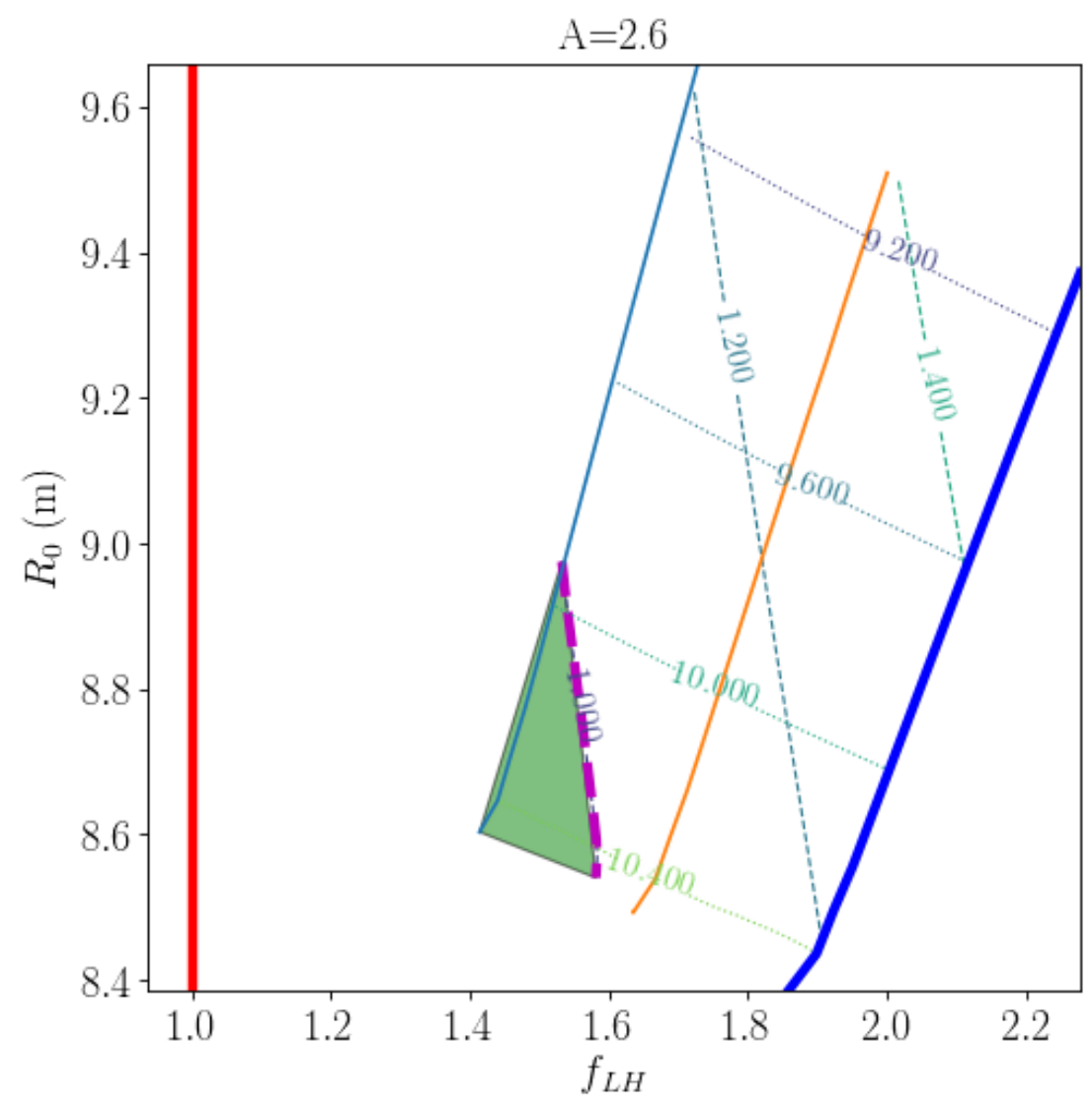

Figure 3. Operating space in $R$ and $f_{L H}$ as derived using the PROCESS code. The aspect ratio and corresponding elongation have been varied from $\mathrm{A}=3.6$ (upper plot), $\mathrm{A}=3.1$ (middle plot), $\mathrm{A}=2.6$ (lower plot). The $P_{\text {sep }} B_{T} / q_{95} A R$ range has been shown using solid lines. The legend is the same for all three plots and the $P_{\text {sep }} B_{T} / q_{95} A R=9 \mathrm{MW} \mathrm{T} / \mathrm{m}^{2}$ has been highlighted for comparison with Fig.2. The contours of the normalised Reinke scaling is depicted with dashed lines. The $c_{Z, \text { det }}=1.0$ value has again been highlighted for easier comparison with Fig.2. The maximum magnetic field on the TF coil is denoted by dotted contour lines, as this becomes an engineering limit for high aspect ratios.

At high aspect ratio, no operational domain with $\hat{c}_{Z, \text { det }} \leq 1.0$, a maximum field on the TF coil $<12 \mathrm{~T}$ (limited by structural strength) and $P_{s e p} B_{T} / q_{95} A R \geq 6.5 \mathrm{MW} \mathrm{T/m}$ can be identified. In contrast, at low aspect ratio there is scope to operate above $f_{L H}=1.4$ and the magnetic field on the coils is no longer a stringent limit. The device size is in this case set by the need to fit components into the radial build. This provides considerably more flexibility for the choice of operating point.

It is worth however to recall that the Goldston scaling foresees a weaker dependence of $\lambda_{q}$ on the aspect ratio, as previously discussed. Thus, the benefit appearing here by adopting a lower aspect ratio might in reality be less pronounced, at least concerning $P_{s e p} B_{T} / q_{95} A R$.

\section{Consequences beyond EU-DEMO}

In the previous sections, the analysis has been focused on the EU-DEMO machine, and for this reason the condition of constant fusion power was imposed. It is interesting to relax such a constraint and try to draw some conclusions which are valid on a more general level. To do this, $R$ and $B_{T}$ are now considered as independent variables. The Reinke criterion expressed in terms of $R, B_{T}$ and $f_{L H}$ reads therefore

$$
\hat{c}_{Z, d e t} \propto f_{L H}^{1.14} B_{T}^{0.88} R^{1.33}
$$

whereas (employing Eq.24)

$$
\frac{P_{s e p} B_{T}}{q_{95} A R} \propto f_{L H} B_{T}^{2.52} R^{0.16}
$$


Here, the role of $\kappa$ and $A$, investigated with PROCESS, is again neglected. As one can observe by looking at the exponents in Eq.34 and 35, an analogous conclusion to the constant fusion power case can be drawn, namely:

- The Reinke criterion has the effect of limiting the allowable size of the tokamak, as its dependence on $R$ is stronger than the one on $B_{T}$. This implies that small high-field devices require a lower impurity concentration to achieve detachment.

- $\quad P_{\text {sep }} B_{T} / q_{95} A R$ on the contrary is very weakly dependent on $R$, i.e. the limit on the heat flux by reattachment is essentially equivalent to a limit on the maximum field which is allowed in the design of a tokamak. Clearly, the maximum tolerable heat flux depends crucially on the technical solutions available, potentially becoming infinite if a configuration able to always maintain detachment is found.

Eq.35 suggests a close correlation between the maximum heat flux which can be withstood by the chosen divertor technology and the acceptable toroidal magnetic field. This means that compact devices with high magnetic field are much more susceptible with respect to the problem of reattachment than large devices with moderate field, but conversely they are less critical for the attainment of detachment via seeded impurities. Thus, they require a technological solution that can either deal with very large heat fluxes or with a divertor configuration that can ensure, under any circumstance, the maintenance of the detached conditions.

Also, Eq.34 suggest that large fusion power plants with moderate field and with a fusion power level exceeding EU$\mathrm{DEMO}$, thus needing a larger major radius, will be very challenging with respect to the maintenance of detachment via seeded impurities. This is consistent with the conclusions of the more comprehensive investigation presented in [11]. It is also important to recall that the figures of merit analysed in this manuscript, and concerning the divertor protection, are not the only aspect limiting the viable design space. Thus, in order to conclude on the feasibility of a given reactor design point, other technological constraints had to be taken into account.

\section{Conclusions}

In this paper, the preliminary design criteria to ensure divertor protection for the EU-DEMO - a 2 GW fusion power reactor operated in $\mathrm{H}$-mode with a conventional, lower single null divertor employing seeded impurity for power dissipation - have been discussed. Corresponding figures of merit to quantitatively compare different design solutions in terms of $R$ and $B_{T}$ have been identified in the existing literature, and explored. The Reinke criterion [34] has been utilised to estimate the necessary concentration of seeded impurities to detach the divertor, while $P_{\text {sep }} B_{T} / q_{95} A R$ is utilised as a proxy for the magnitude of the heat flux on the divertor plate in the case of plasma reattachment. By means of a simple $0 \mathrm{D}$ argument it has been shown that the two figures of merit possess opposite dependences on the major radius and on the toroidal magnetic field when the fusion power is kept constant. This implies the existence, for EU-DEMO, of both a maximum radius and a maximum magnetic field above which the divertor compatibility cannot be ensured - either because of a too high impurity concentration required to detach the divertor or because of a too high heat flux by reattachment, which cannot be dealt with by the available heat flux removal technology, although it has to be acknowledged that, by virtue of the simplicity of the model and of the various approximation adopted, the present analysis is not adequate to provide an accurate determination of the limiting $R$ and $B_{T}$ values, but only to capture their effect on the divertor compatibility problem. As already shown in [11], there will be no solution for a $\sim 1 \mathrm{GW}$ electric power LTS device with $A=3.1$ with a conventional divertor and seeded impurities. Other divertor solutions which are able to ensure that a loss of detachment never occur, might then make high field machine more attractive - provided that their integrability in a reactor is possible.

Furthermore, an attempt to extend the consequences of this EU-DEMO related analysis to a more general level has been undertaken. The main conclusion is that, generally speaking, the strong dependency of $P_{s e p} B_{T} / q_{95} A R$ on the magnetic field strength hampers the realisation of compact devices with very high field, which would then require either the development of a technological solution to withstand extremely high heat flux during the transients or a divertor concept able to ensure that reattachment never occurs. On the contrary, large devices are limited in size and thus in power by the necessary concentration of impurity to attain detachment, threatening the stability, or the performance, of the discharge.

\section{Acknowledgments}

Enlightening discussions with P. Barabaschi, M. Wischmeier, A. Loarte, J. H. You and K. Lackner are gratefully acknowledged by the authors. This work has been carried out within the framework of the EUROfusion Consortium and has received funding from the Euratom research and training programme 2014-2018 under grant agreement No 633053. The views and opinions expressed herein do not necessarily reflect those of the European Commission. 


\section{References}

[1] Federici G. et al., 2017 Int. Symp. on Fusion Nuclear technology (ISFNT), Kyoto, Japan

[2] Uckan N.A. 1989 ITER physics design guidelines, ITERTN-PH-8-6 (http://www.iaea.org/inis/collection/NCLCollectionStore/_Public/21/068/21068960.pdf?r=1)

[3] Romanelli F. et al 2012 A roadmap to the realisation of fusion energy, EFDA Document

[4] Federici G. et al., accepted by Nucl. Fusion - IAEA FEC 2018

[5] Wagner F et al., 1982 Phys. Rev. Lett. 491408

[6] Martin Y.R. et al., 2008 J. Phys.: Conf. Ser. 123012033

[7] Boucher D. et al., 1996 ITER Physics Design Description Document, p.2-2

[8] Snipes J. A., 1997 ITER H-mode Threshold Database Working Group, Controlled Fusion and Plasma Physics, $24^{\text {th }}$ EPS Conference, Berchtesgaden, 21-A, p.III, 961

[9] Wenninger R.et al., 2015 Nucl. Fusion 55063003

[10] Wenninger R. et al., 2017 Nucl. Fusion 57046002

[11] Siccinio M. et al., 2018 Nucl. Fusion 58016032

[12] Kovari M. et al., 2014 Fusion Eng. Des. 893054

[13] Kovari M. et al., 2016 Fusion Eng. Des. 1049

[14] Maviglia F. et al., 2018 Fusion Eng. Des, 136410

[15] Bachmann C. et al., accepted by Fusion Eng. Des.

[16] Maviglia F. et al., 2018 Fusion Eng. Des. 136, 410

[17] Mattei M., PMI Final Report 2018, unpublished

[18] Siccinio M. et al., submitted to Nucl. Fusion (IAEA FEC 2018)

[19] Evtikhin V.A. et al., 2002 Plasma Phys. Control. Fusion 44, 955

[20] Pitts R. et al., 2011 Jour. Nucl. Mat. 4155957

[21] Albanese R., Ambrosino R. and Mattei M., 2015 Fus. Eng. Des. 96664

[22] You J.H. et al., 2016 Nuclear Materials and Energy 9171

[23] Makowski M., 2012 Phys. Plasmas 19056122

[24] Wenninger R. et al., 2014 Nucl. Fusion 54, 114003

[25] Eich T. et al., 2011 Phys. Rev. Lett. 107215001

[26] Eich T. et al., 2013 Nucl. Fusion 53093031

[27] Chang C. S. et al., 2017 Nucl. Fusion 57116023

[28] Bernert M. et al., 2017 Nucl. Mat. Energy 12111

[29] Zohm H. et al 2017 Nucl. Fusion 57086002

[30] Shimada M. et al., Heat and Nuclear Load Specifications for ITER

[31] Raffray A.R.and Federici G., 1997 J. Nucl. Mater. 24485

[32] Maviglia F. et al., 2016 Fusion Eng. Des. 1091067

[33] Kallenbach A. et al., 2013 Plasma Phys. Control. Fusion 55124041

[34] Reinke M.L. 2017 Nucl. Fusion 57034004

[35] Goldston R. J., Reinke M. L. and Schwartz J. A., 2017 Plasma Phys. Control. Fusion 59055015

[36] Subba F. et al., 2018 Plasma Phys. Control. Fusion 60035013

[37] Zohm H., 2010 Fusion Science and Technology 58:2 613

[38] Lux H. et al., 2016 Plasma Phys Control. Fusion 58, 7, 075001

[39] Lux H. et al., 2015 Fus. Eng. Des. 10142

[40] Ward, D. J., 2010 Plasma Phys. Control. Fusion 52, 124033

[41] Reinke, M. L. et al., 2011 Journ. Nucl. Mat. 415, S340

[42] Utoh H. et al., 2018 Fus. Eng. Des. 136 B 874 